\title{
NUMERICAL SIMULATION OF THE CONFLUENCE FLOW BY USING k- $\varepsilon$ MODELS
}

\author{
By Liang CHENG", Saburo KOMURA ${ }^{* *}$ and Ichiro FUJITA ${ }^{* . *}$
}

\begin{abstract}
The two-dimensional T-type confluence flow is numerically simulated by using $\mathrm{k}-\varepsilon$ turbulence models. It is found that standard $\mathrm{K}-\varepsilon$ turbulence model reveals severe discrepancies in predicting the confluence flow which is characterized by a large recirculating region. To analyze the reasons of the poor prediction, further studies are conducted by means of the low-Rynolds number version of $k-\varepsilon$ model and an improved $k-\varepsilon$ model proposed by Hanjalic and Launder for decelerated flows. By comparing the numerical results with the experimental results, it is understood that the wall functions used in the standard $k-\varepsilon$ turbulence model and the inaccurate modeling of $\varepsilon$-equation are two main reasons for the poor predictions. Finally, some suggestions are proposed for the numerical simulations of the confluence flow.
\end{abstract}

Keywords: confluence flow, $k-\varepsilon$ model, recirculation region

\section{INTRODUCTION}

Confluence flow is often encountered in the engineering. To estimate the flow influence on channel scour and sidewall erosion, or to compute the concentration rate of contamination and local sedimentation process of river confluence, the detailed knowledge about the flow is of great fundamental and practical importance. Some studies on confluence flow have been made experimentally and analytically. However, the numerical studies on it have not been well reported until now. The first numerical report on the flow is that of Tamai and Ueda[1]. Tamai and Ueda's simulation is three dimensional one with the standard $k-\varepsilon$ turbulence model. They followed the SIMPLE algorithm and partially parabolic computation procedure of Patrap and Spalding. Recently, the three dimensional computation and depth averaged computation of the confluence flow in generalized coordinates using standard $\mathrm{K}-\varepsilon$ model were reported by Weeraknoon[2]. The models have been applied to the laboratory channel flow and river flows. Generally, it can be observed that smaller recirculating region behind downstream corner is predicted by using standard $k-\varepsilon$ model.

Although the standard $k-\varepsilon$ model has been widely used to simulate many kinds of flow, sometimes it is in seriously error even for relatively simple flows. For examples, recirculating flow and boundary layer flow with adverse pressure gradient are typical two of these. The detailed researches on this have been reported by Hanjalic and Launder[3] and Rodi and Scheuerer[4]. For the confluence flows, the standard $k-\varepsilon$ turbulence model has been used by some investigators, but the appropriateness of the model for the confluence flow is not well investigated. Actually, according to the results obtained by using standard $k-\varepsilon$ model, the predictive ability of the model on confluence flow is questionable. The purpose of the present paper is to examine the model performance on confluence flows and try to get an acceptable prediction on the confluence flow.

\section{BASIC EQUATIONS AND TURBULENCE MODELS}

With the turbulent stresses substituted by eddy-viscosity relations, the equations governing the flow considered are as follows:

$$
\begin{gathered}
\frac{\partial U}{\partial x}+\frac{\partial V}{\partial y}=0 \\
\frac{\partial U}{\partial t}+U \frac{\partial U}{\partial x}+V \frac{\partial U}{\partial y}=-\frac{1}{\rho} \frac{\partial P}{\partial x}+2 \frac{\partial}{\partial x}\left[v_{e} \frac{\partial U}{\partial x}\right]+\frac{\partial}{\partial y}\left[v_{e}\left(\frac{\partial U}{\partial y}+\frac{\partial V}{\partial x}\right)\right]
\end{gathered}
$$

* Dr. of Eng., Lecturer of Civil Eng. Dept., Dalian University of Technology, Dalian 116024, P. R. China

** Member, Dr. of Eng., Prof. of Civil Eng. Dept., Gifu University, Yanagido 1-1, Gifu, Japan

*** Member, Ph.D., Ass. Prof. of Civil Eng. Dept., Gifu University, Yanagido 1-1, Gifu, Japan 


$$
\frac{\partial V}{\partial t}+U \frac{\partial V}{\partial x}+V \frac{\partial V}{\partial y}=-\frac{1}{\rho} \frac{\partial P}{\partial y}+\frac{\partial}{\partial x}\left[v_{e}\left(\frac{\partial U}{\partial y}+\frac{\partial V}{\partial x}\right)\right]+2 \frac{\partial}{\partial y}\left[v_{e} \frac{\partial V}{\partial y}\right]
$$

where $U$ and $V$ are the mean velocities in $x$ and $y$-direction, respectively, $P$ is the pressure, $v$ is the kinematic fluid viscosity, $v_{t}$ the eddy viscosity, $v_{e}=v+v_{t}$ and $\rho$ is the density.

In present paper, calculations have been carried out with the following turbulence models: the standard $k-\varepsilon$ model, the low-Reynolds number version of the $k-\varepsilon$ model by Jones and Launder[5], denoted JL, and the improved $k-\varepsilon$ model for adverse pressure gradient proposed by Hanjalic and Launder[3], denoted HL.

The standard $k-\varepsilon$ model and its two variations used in this paper employ the eddy viscosity concept and calculate the eddy viscosity $v_{1}$ from

$$
v_{t}=C_{\mu} \frac{k^{2}}{\epsilon}
$$

where $\mathrm{k}$ is the turbulence energy and $\varepsilon$ is the dissipation rate. The distribution of $\mathrm{k}$ and $\varepsilon$ is determined by solving the transport equations which, in the standard $k-\varepsilon$ model, are as follows:

$$
\begin{aligned}
& \frac{\partial k}{\partial t}+U \frac{\partial k}{\partial x}+V \frac{\partial k}{\partial y}=\frac{\partial}{\partial x}\left(\left(v+\frac{v_{t}}{\sigma_{k}}\right) \frac{\partial k}{\partial x}\right)+\frac{\partial}{\partial y}\left(\left(v+\frac{v_{t}}{\sigma_{k}}\right) \frac{\partial k}{\partial y}\right)+P_{k}-\epsilon \\
& \frac{\partial \epsilon}{\partial t}+U \frac{\partial \epsilon}{\partial x}+V \frac{\partial \varepsilon}{\partial y}=\frac{\partial}{\partial x}\left(\left(v+\frac{v_{t}}{\sigma_{e}}\right) \frac{\partial \epsilon}{\partial x}\right)+\frac{\partial}{\partial y}\left(\left(v+\frac{v_{t}}{\sigma_{e}}\right) \frac{\partial \epsilon}{\partial y}\right)+\frac{C_{1} \epsilon}{k} P_{k}-\frac{C_{2} \epsilon^{2}}{k} \\
& \text { where } \quad P_{k}=v_{e}\left(2\left(\left(\frac{\partial U}{\partial x}\right)^{2}+\left(\frac{\partial V}{\partial y}\right)^{2}\right)+\left(\frac{\partial U}{\partial y}+\frac{\partial V}{\partial x}\right)^{2}\right)
\end{aligned}
$$

represents the production of $\mathrm{k}$ by the interaction of mean velocity gradients and turbulent stresses. The constants take the values as: $C_{\mu}=0.09, C_{1}=1.44, C_{2}=1.92, \sigma_{k}=1.0$ and $\sigma_{\varepsilon}=1.3$.

The low-Reynolds number $\mathrm{k}-\varepsilon$ model(JL) resolves the flow field down to the wall and hence accounts directly for viscous effects. To this end the empirical constants $C_{\mu}, C_{1}$ and $C_{2}$ used in standard $k-\varepsilon$ model are multiplied by functions $f_{\mu}, f_{1}, f_{2}$ which involve the molecular viscosity. The extra terms $D, E$ are added to the transport equation of $k$ and $\varepsilon$, respectively, only for the computational reason. The exact formulae of the model are expressed as follows[5]:

$$
\begin{aligned}
& \frac{\partial k}{\partial t}+U \frac{\partial k}{\partial x}+V \frac{\partial k}{\partial y}=\frac{\partial}{\partial x}\left(\left(v+\frac{v_{t}}{\sigma_{k}}\right) \frac{\partial k}{\partial x}\right)+\frac{\partial}{\partial y}\left(\left(v+\frac{v_{t}}{\sigma_{k}}\right) \frac{\partial k}{\partial y}\right)+P_{k}-\epsilon+D \\
& \frac{\partial \varepsilon}{\partial t}+U \frac{\partial \varepsilon}{\partial x}+V \frac{\partial \varepsilon}{\partial y}=\frac{\partial}{\partial x}\left(\left(v+\frac{v_{t}}{\sigma_{e}}\right) \frac{\partial \epsilon}{\partial x}\right)+\frac{\partial}{\partial y}\left(\left(v+\frac{v_{t}}{\sigma_{e}}\right) \frac{\partial \varepsilon}{\partial y}\right)+ \\
& \frac{C_{1} f_{1} \epsilon}{k} P_{k}-\frac{C_{2} f_{2} \varepsilon^{2}}{k}+E \\
& f_{1}=1.0 \quad, \quad f_{2}=1.0-0.3 \exp \left(-R_{T}^{2}\right) \\
& D=-2 v\left(\frac{\partial \sqrt{k}}{\partial y}\right)^{2}, \quad E=2 v v_{t}\left(\frac{\partial^{2} \bar{U}}{\partial y^{2}}\right)^{2}
\end{aligned}
$$

and $R_{T}=k^{2} / \mathrm{ev}$ is turbulence Reynols number.

The empirical constants of JL model are given the values as: $C_{\mu}=0.09, C_{1}=1.45, C_{2}=2.0, \sigma_{k}=1.0$ and $\sigma_{\varepsilon}=1.3$.

With the intention to improve the $k-\varepsilon$ model when applied to recirculating flows, Hanjalic and Launder[3] proposed a modified $k-\varepsilon(\mathrm{HL})$ for decelerated flows. The modification lies in the production term $P_{e}$ in equation (6). The modified $\varepsilon$-equation reads 


$$
\begin{aligned}
\frac{\partial U}{\partial t}+U \frac{\partial \epsilon}{\partial x}+V \frac{\partial \epsilon}{\partial y}=\frac{\partial}{\partial x}\left(\left(v+\frac{v_{t}}{\sigma_{e}}\right) \frac{\partial \epsilon}{\partial x}\right)+\frac{\partial}{\partial y}\left(\left(v+\frac{v_{t}}{\sigma_{e}}\right) \frac{\partial \epsilon}{\partial y}\right)-C_{1} \frac{\epsilon}{k} \overline{u v} \frac{\partial U}{\partial y} \\
-C_{3}\left(\overline{u^{2}}-\overline{v^{2}}\right) \frac{\partial U}{\partial x}-C_{2} \frac{\epsilon^{2}}{k}
\end{aligned}
$$

where $\overline{u^{2}}, \overline{v^{2}}$ and $\overline{u V}$ are stresses, $C_{3}$ is the empirical constant and $C_{3}=4.44$ given by Hanjalic and Launder[3].

The computations conducted in present paper employ the following boundary conditions: for the inlet boundaries of both main and tributary channels, fully developed distributions of turbulence flow are applied; for the outlet boundary, taking the boundary far enough from the region of interest, it can be assumed that continuative condition for all variables is satisfied; for the wall boundaries, when the standard $k-\varepsilon$ model is used, the viscous near wall zone is bridged by wall functions, when JL model is applied, the no-slip-condition is employed at the wall.

The finite difference scheme used in present study is that of VOF proposed by Hirt and Nichols[6].

\section{APPLICATIONS AND DISCUSSIONS}

Present research is focused on the appropriateness of $k-\varepsilon$ model to the confluence. So only two dimensional T-type confluence flow in a duct is studied. The calculations are performed in dimensionless form. The averaged outlet velocity $U_{0}$ of the main channel, main channel width $B_{0}$ and the kinematic viscosity $v$ are taken as the characteristic quantities in the dimensionless processing. All the computations are carried out under the condition that the discharge ratio of the branch to the total discharge is 0.6 , the width ratio of the branch to the main channel is $2 / 3$ and the Reynolds number $\mathrm{Re}$ is 98000 . The experimental velocity distribution was measured under the same conditions mentioned above by using velocity propeller.

Fig. 1 gives the averaged velocity vector distribution by the experiment. The experiment was conducted in the open channel. The velocity distribution given in Fig. 1 was measured horizontally at the position $\mathrm{z} / \mathrm{H}_{3}=0.9$, where $\mathrm{H}_{3}(=5.0 \mathrm{~cm})$ is the downstream water depth of the main channel and $\mathrm{z}$ is the upward coordinate with the origin at flat channel bottom. Fig.2 shows the corresponding numerical result by the standard $\mathrm{K}-\varepsilon$ model. It can be observed that the predicted recirculation region length( which is defined as the length from the downstream corner of the confluence to the reattachment point of the flow) $\mathrm{L}=0.65$ is much smaller comparing with the experimental result $L=2.2$. Obviously, this is not a good prediction of the flow. To make a better prediction, the reasons for the poor prediction have to be understood. By considering the characteristics of the confluence flow and the standard K- $\varepsilon$ model, one may infer that the wall functions used in the model and the possible adverse pressure gradient of the flow along the downstream right bank of the channel may be the two main factors which are responsible for the poor prediction. As we know, the wall functions used in the standard $k-\varepsilon$ model have been deduced under the condition that flow does not separate. Many researches have shown that recirculation flow can not be well represented by using the wall functions. On the other hand, the confluence flow is mainly characterized by the recirculation. To prove this, the same simulation is performed by means of the JL model which allow the computation down to the wall boundary directly. The computational recirculation region length $\mathrm{L}=1.30$ (as shown in Fig.3) is much better than that of standard $\mathrm{k}-\varepsilon$ model, but is still not satisfactory comparing with the experimental result $(\mathrm{L}=2.2)$. That is to say, the wall function is not the only factor responsible for the poor prediction.

Hanjalic [3] and Rodi [4] have pointed out that the standard K- $\varepsilon$ model can not predict the flows with adverse pressure distributions correctly because the set of empirical coefficients in the $\varepsilon$-equation, which have been determined by referring to zero pressure gradient boundary layers, are not compatible with the experimental observations in decelerated flows. In the case of the confluence flow studied in this paper, although the pressure distribution has not been measured physically, the numerical result obtained by standard $\mathrm{K}-\varepsilon$ model shows that there exists a strong adverse pressure distribution along the downstream right bank of the channel, as shown in Fig.4, in which $\mathrm{P}_{0}$ is the reference pressure at the outlet section. It should be noticed that whether this adverse pressure gradient exists physically in the real flow or not is unimportant, the important point is that this numerical gradient may distorts the prediction as the real one does. To prove this, the improved $\varepsilon$-equation for the adverse pressure distribution flows proposed by Hanjalic and Launder[3] is incorporated into both of the standard model and $\mathrm{JL}$ model and the computations are conducted by using the 
two models. The computation results are shown in Fig.5 and Fig.6, respectively. The predicted recirculation region length $\mathrm{L}=1.46$ by the standard model with improved $\varepsilon$-equation is better than that of the standard model, $\mathrm{L}=\mathbf{0 . 6 5}$. The strong effect of the adverse pressure gradient can be seen clearly from this comparison.

The result $\mathrm{L}=1.78$ by the $\mathrm{JL}$ model with improved $\varepsilon$-equation can be considered as that it has excluded the effects of both wall functions and the adverse pressure distribution. Therefore, it is still smaller than the experimental result $\mathrm{L}=2.2$ and hard to be accepted as a good prediction on the flow. The reasons for that are still not very clear. However, the following aspects may impose some influences on the predictions in present research:

(1) the finite difference used in the present paper is a hybrid of the upwind and second order central schemes. Many researchers have reported that this kind scheme usually introduces too much numerical diffusion into the numerical solution and the numerical results can be distorted severely by this numerical diffusion;

(2) the turbulence models used in the present paper can not take account the streamline curvature into the model, whereas streamline curvature effect is very strong in the confluence flows;

(3) the present simulation is a two dimensional one while the experiment was the three dimensional one.

The research on these aspects is not included in this paper and will be presented later on. It is hoped that some improvements can be achieved by incorporating these aspects into the simulations. According to the research presented here, the following conclusions can be obtained:

1. The standard $k-\varepsilon$ turbulence model is not appropriate to the confluence flow with recirculating region because of the modelled $\varepsilon$-equation and the wall functions used in the model;

2. The Hanjalic and Launder's improved $\mathrm{k}-\varepsilon$ turbulence model can give an improved prediction on the confluence flow but not quit satisfactory one;

3. Further researches on confluence flow are needed because some aspects of the flow and turbulence model behavior have not been well understood.

\section{REFERENCES}

1. Tamai, N., and Ueda, S., Proc. 43rd Annual Conf. of JSCE, 442-443, 1987.

2. Weerakoon, S. B., Ph.D. Thesis, Tokyo University, Japan, 1990.

3. Hanjalic, K., and Launder, B. E., ASME J. of Fluid Eng., Vol.102, P.34, 1980.

4. Rodi, W., and Scheuerer, G., Transactions of the ASME, Vol.108, P.174, 1986.

5. Jones, W.P., and Launder, B.E., International Journal of Heat and Mass Transfer, Vol.16, P.1119, 1973.

6. Hirt, C.W., and Nichols, B.D., J. Comput. Phys., Vol.39, P.201, 1981. 


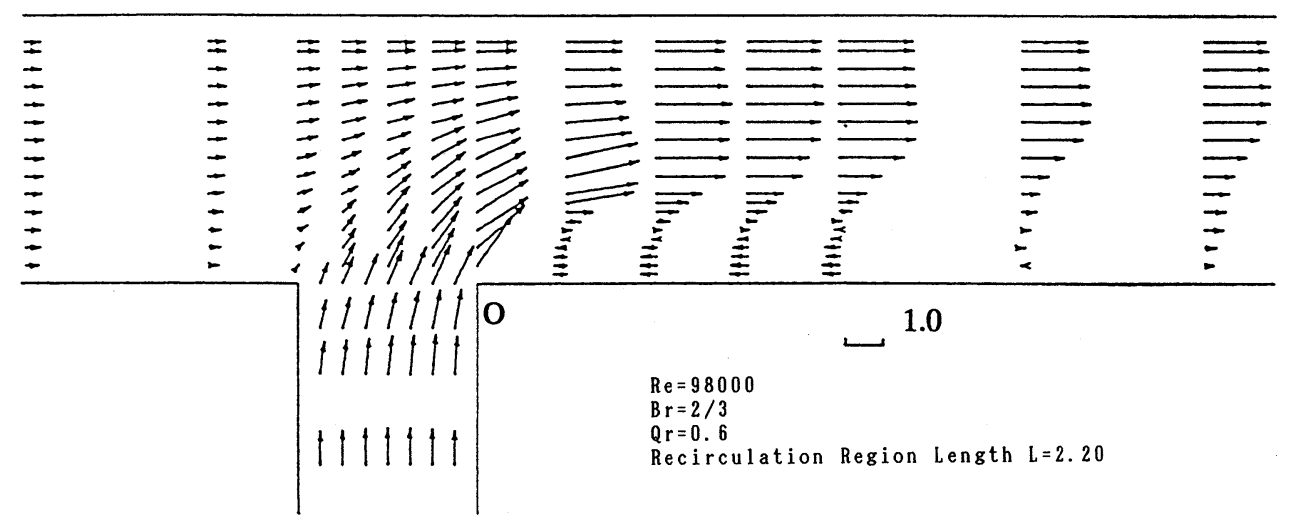

Fig.1 Experimental velocity distribution by propeller

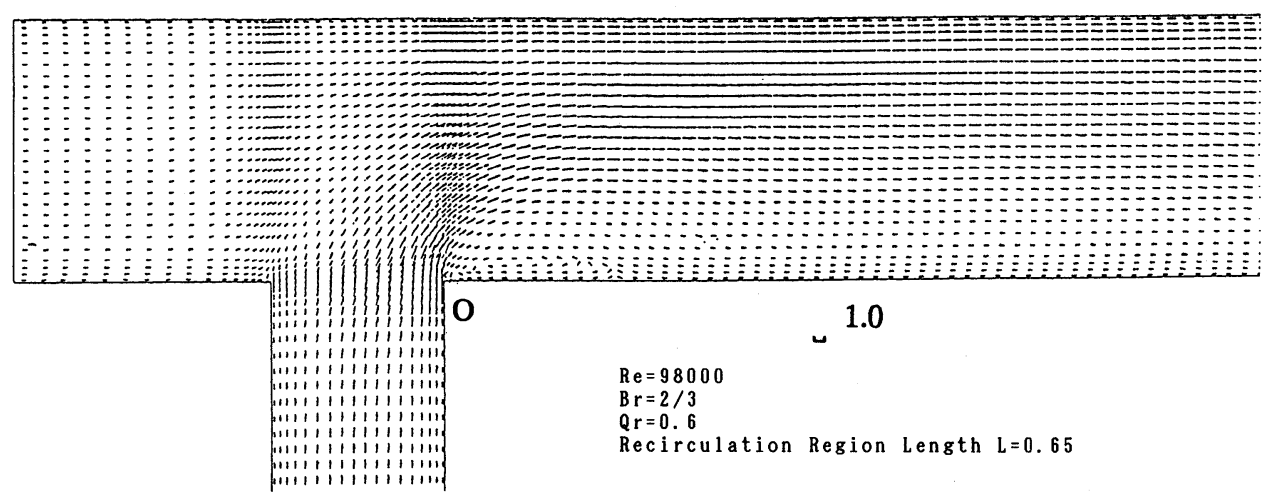

Fig.2 Numerical velocity distribution by the standard $k-\varepsilon$ model

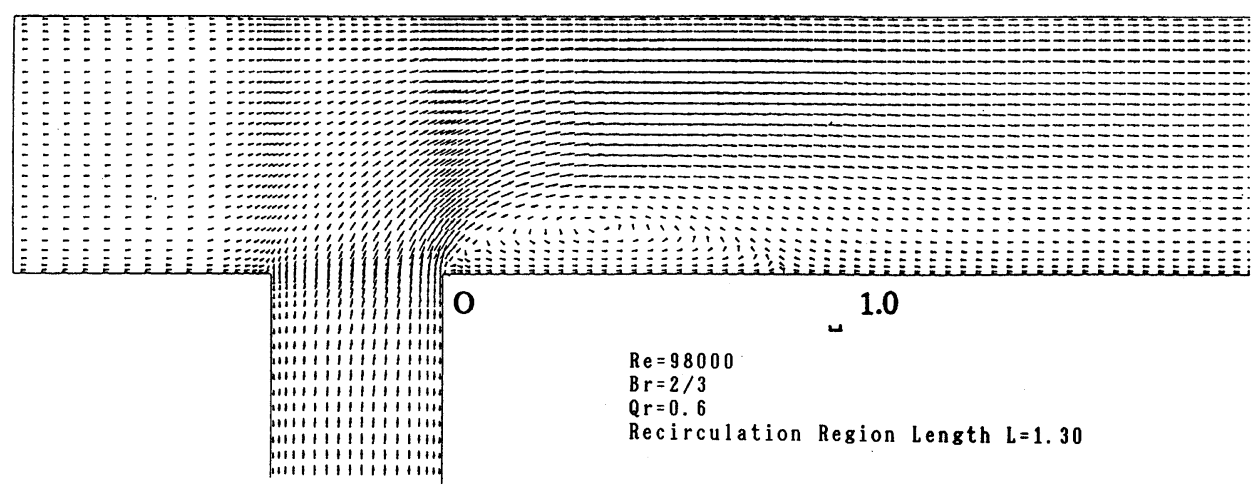

Fig.3 Numerical velocity distribution by JL $k-\varepsilon$ model 


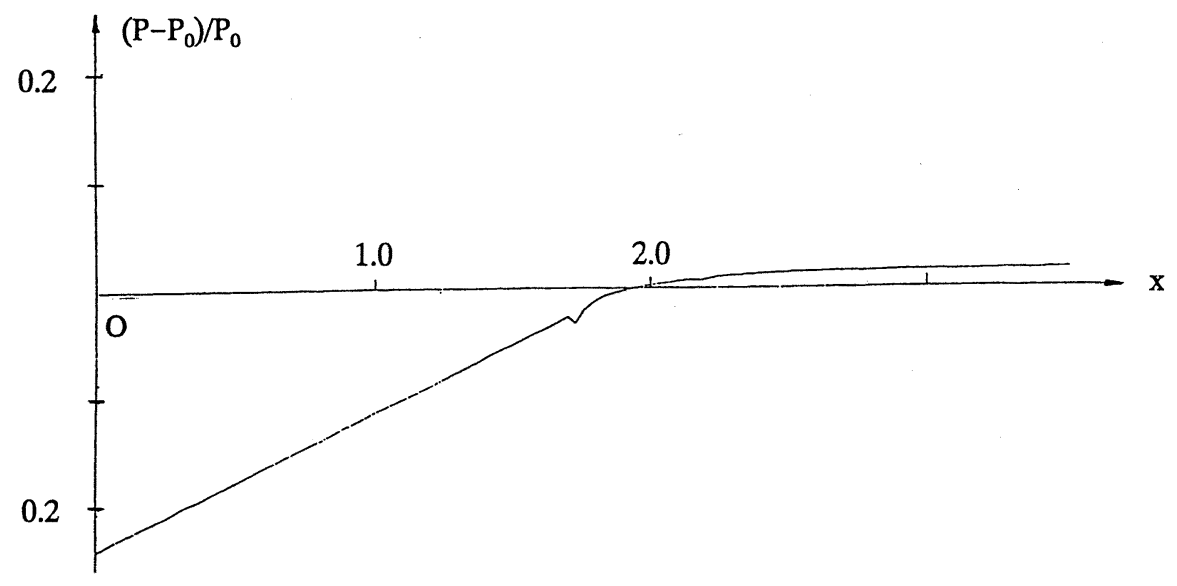

Fig.4 Numerical pressure distribution by the standard $k-\varepsilon$ model

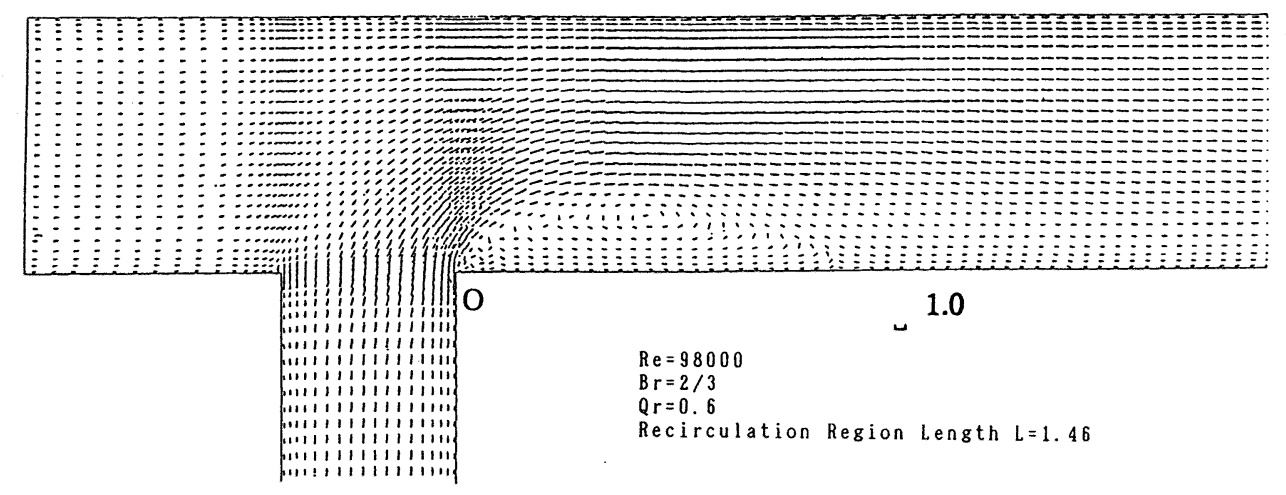

Fig.5 Numerical velocity distribution by the standard $k-\varepsilon$ model with modified $\varepsilon$-equation

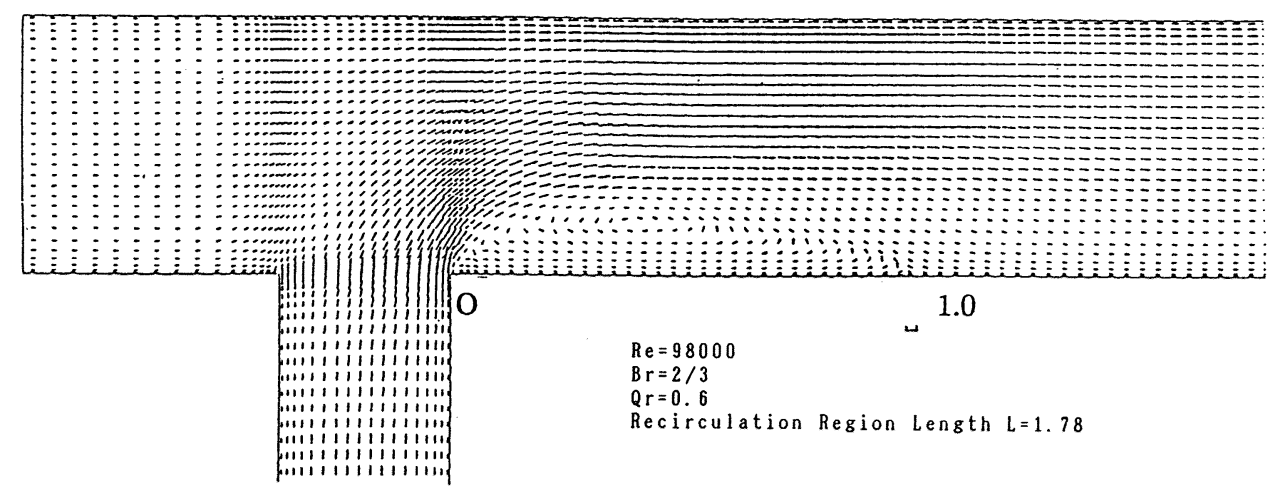

Fig.6 Numerical velocity distribution by JL $k-\varepsilon$ model with modified $\varepsilon$-equation 